Claudio DE BONI, Storia di un'utopia - La religione dell'Umanità di Comte e la sua circolazione nel mondo

\title{
Annie Petit
}

\section{OpenEdition}

1 Journals

Édition électronique

URL : http://journals.openedition.org/ress/3171

DOI : 10.4000/ress.3171

ISBN : $1663-4446$

ISSN : 1663-4446

Éditeur

Librairie Droz

Édition imprimée

Date de publication : 15 novembre 2015

Pagination : 279-281

ISSN : 0048-8046

Référence électronique

Annie Petit, «Claudio DE BONI, Storia di un'utopia - La religione dell'Umanità di Comte e la sua circolazione nel mondo ", Revue européenne des sciences sociales [En ligne], 53-2 | 2015, mis en ligne le 15 novembre 2015, consulté le 22 septembre 2020. URL : http://journals.openedition.org/ress/3171 ; DOl : https://doi.org/10.4000/ress.3171

Ce document a été généré automatiquement le 22 septembre 2020.

(c) Librairie Droz 


\title{
Claudio DE BONI, Storia di un'utopia- La religione dell'Umanità di Comte e la sua circolazione nel mondo
}

\author{
Annie Petit
}

\section{RÉFÉRENCE}

Claudio DE BONI, 2013, Storia di un'utopia - La religione dell'Umanità di Comte e la sua circolazione nel mondo, Milano, Mimesis, « Diacronie », 446 p.

1 Cet ouvrage fait le point sur la diffusion d'un aspect du positivisme comtien, qui n'a pas été l'aspect privilégié par les études qui en ont été faites, en tout cas dans la plupart des travaux académiques français, voire européens. La fondation par Auguste Comte d'une nouvelle religion, la «religion de l'Humanité »-dont les rites sont expressément décalqués de rites catholiques -, a soulevé dès le xix ${ }^{\mathrm{e}}$ siècle bien des réserves, y compris de la part de penseurs qui, quoique ayant souscrit à la philosophie positive, ont vu dans les formes religieuses du positivisme une déviance regrettable, à l'instar d'Émile Littré ou de John Stuart Mill. Il reste qu'une telle déviance fut davantage imputée à un épisode personnel de la vie d'Auguste Comte qu'à la cohérence philosophique de sa pensée. En outre, le projet même de fonder une religion est apparu comme une entreprise désuète, liée aux tendances romantiques et utopiques assez communément partagées au XIX ${ }^{e}$ siècle. De ce fait, l'intérêt pour le positivisme en tant que philosophie des sciences et de la connaissance s'est très vite dégagé des considérations religieuses que Comte associait à sa «sociologie » et dont il tira sa "politique positive». Et il s'ensuit que les néo-positivismes n'ont pas retenu non plus ces aspects du positivisme comtien.

Pourtant le livre de Claudio De Boni montre combien le projet d'une «religion de l'Humanité » a passionné et a été riche d'influences et d'héritages féconds. De fait, le projet comtien soulève des problématiques aussi capitales - et toujours d'actualité - 
que la question de la distribution des pouvoirs, le lien de la politique et de la morale, le souci primordial de l'éducation, l'inscription dans l'horizon historique, la conception des rapports entre différentes composantes sociales, l'articulation du collectif et de l'individuel, etc., en sorte que l'auteur est fondé à parler des «incidences internationales du phénomène » (p. 9).

3 Claudio De Boni a justement choisi pour son étude de suivre le rayonnement géographique de la "religion de l'Humanité » d'Auguste Comte. Au préalable, le chapitre 1 en rappelle la genèse et ses principaux thèmes: dans une Humanité vue comme Grand-Être, il souligne la continuité sociale du phénomène en mettant en exergue ses deux attributs fondamentaux que sont la «fixité principale» et la "perfectibilité secondaire» (p. 19); puis il défend une conception de la politique comme « moralisation de la société » (p. 24). Le chapitre 2 analyse l'héritage français en rendant compte des tensions que la confusion du politique et du religieux provoque parmi les disciples. Puis le chapitre 3 s'intéresse plus spécialement aux répercussions des thèses comtiennes en Grande-Bretagne, où la part faite au religieux dans son rapport à la Science fait l'objet de vives discussions et entraîne des écoles concurrentes (p. 235 et suiv.). Les chapitres 4 et 5 étendent les analyses aux débats qui ont touché les pays plus lointains, l'Inde et les États-Unis d'Amérique mais également de nombreux pays d'Amérique latine - Argentine, Uruguay, Chili, Venezuela, et surtout Mexique et Brésil - où le souci de l'universel manifesté par le positivisme et la "religion de l'Humanité " prend un sens particulier dans le contexte colonial et le rapport aux peuples indigènes.

4 Dans chacun des chapitres, Claudio De Boni ordonne ses analyses selon les positions des différents auteurs qu'il examine. Et l'on peut apprécier la richesse de la documentation sur laquelle il s'appuie, tout comme la clarté des synthèses qu'il en présente. L'auteur sait mettre en perspective les positions des uns et des autres, non seulement par rapport aux thèmes clés de la religion de l'Humanité de Comte, mais aussi en confrontant les décalages que chacun y apporte.

5 Ainsi, chez les Français, Claudio De Boni analyse avec finesse comment Littré, concevant la sociologie essentiellement comme science de l'histoire, subvertit les visées comtiennes en tirant, notamment, le positivisme vers le socialisme. Du côté de Laffitte et du groupe "orthodoxe", il rend compte de la tension entre le souci de diffusion scientifique des uns, conduisant à une action centrée sur l'instruction et l'éducation, et le militantisme religieux des autres, sans oublier l'inspiration prolétarienne de ceux qui se soucient davantage des questions économiques et politiques concrètes, telles l'organisation du travail ou le syndicalisme. Montrant comment les nombreux médecins adeptes de la nouvelle religion se donnent pour mission de remédier à la «maladie sociale », Claudio De Boni y voit une inflexion du discours philosophique et politique en un discours moral. Et il s'attache à démêler l'écheveau très complexe des différentes tendances, eugéniques ou nationalistes, notamment, à travers lesquelles la pensée comtienne trouve à se diffuser. Il faut à cet égard saluer la précision et la finesse avec lesquelles Claudio De Boni conduit ses analyses, au bonheur de rapprochements qui ne cèdent jamais à la confusion.

6 Ces qualités se retrouvent dans la présentation du positivisme tel qu'il s'est développé en Grande-Bretagne. Claudio De Boni rappelle que dans un milieu formé à la «natural philosophy " où les questions religieuses sont particulièrement sensibles, les thèses comtiennes ont subi d'importants remaniements. Bien sûr, sont présentées les grandes 
figures du militantisme positiviste: John Stuart Mill, George Henry Lewes, Richard Congreve, Frederic Harrison, Harriet Martineau, etc., et les ruptures et scissions qui l'ont marqué. C'est d'ailleurs l'occasion pour Claudio De Boni de proposer d'excellentes synthèses sur la base d'une littérature critique plutôt abondante sur ces sujets. Ainsi, peut-on repérer très clairement les groupes qui se font et se défont, à travers les réseaux des revues où s'expriment les uns et les autres. Et l'on apprécie tout particulièrement le soin apporté à dater les interventions originales, quand bien même elles seraient reprises dans des recueils ultérieurs. Ce chapitre est aussi l'occasion de brosser un tableau beaucoup plus complet du milieu britannique, avec ses nombreux débats sur les Trade Unions, le mouvement fabien, l'ingérence dans les guerres de nationalités, le colonialisme, les polémiques entre science et religion, les rapports à la libre-pensée et à l'agnosticisme, etc. Les mises au point sur l'importance des thèses "évolutionnistes", celles de Thomas Huxley comme celles de Herbert Spencer, sont également remarquables.

7 Les études sur les échos plus lointains des débats qui se jouent autour de la « religion de l'Humanité » allient aussi richesse, clarté et finesse des analyses. Claudio De Boni prend bien soin de préciser les différents points d'adhésion et de rupture, tout en s'attachant à rendre la "couleur locale» des débats. En Inde, par exemple, ils s'inscrivent par rapport à la question des castes, qui implique une réflexion originale sur le problème des élites, ainsi que par rapport aux politiques coloniales. Aux États-Unis, ils gagnent une société déjà agitée par des sectes utopiques et religieuses, sensible au problème du mélange des races, et qui essaie d'articuler le capitalisme et la morale, tout en conciliant souci du collectif et de l'individuel. En Amérique latine, c'est l'histoire politique complexe de ce sous-continent en proie aux guerres civiles qui sert de fond à la réception d'un positivisme d'abord religieux mais dont la vocation politique demeure, dans la mesure où il est diffusé dans les milieux proches du pouvoir ou des oppositions.

8 À lire ces histoires et à les comparer, se dessinent, derrière la variété des modes de transmission et la diversité des enjeux, des lignes forces. L'accueil fait à la « religion de l'Humanité » est l'occasion d'intenses échanges sur des questions qui la dépassent largement. Ainsi est-il partout traité de la question de l'éducation: elle est cruciale pour les positivistes français qui s'appliquent à profiter de la politique d'instruction de la Troisième République; elle ne l'est pas moins pour les britanniques qui choisissent d'appeler leur London Positivist Society, « Positivist School » et illustrent un militantisme soucieux de vulgarisation; elle l'est aussi en Inde, où la théosophe sympathisante Annie Besant participe à la naissance de l'« Université nationale de l'Inde » à Bénarès, et où les positivistes indiens défendent une réorganisation culturelle préalable à toute réorganisation politique; elle l'est enfin en Amérique du Sud où les positivistes, souvent professeurs, sont sinon fondateurs du moins participants actifs à la fondation d'Écoles, d'Instituts ou d'Universités. Dans le domaine plus directement politique, tous reposent la question de la hiérarchie sociale, du partage des pouvoirs, de l'articulation du patriotisme et du nationalisme, du rôle de l'État et de la place de l'individu, de la valeur de la dictature et/ou de la démocratie.

9 Sans doute l'ampleur du domaine concerné par cette étude oblige-t-elle Claudio De Boni à faire des choix très sélectifs dans l'immense documentation explorée: les notes montrent cependant tout le profit qu'on peut encore en tirer. Cet ouvrage est remarquable tant par la qualité de ses synthèses que par la grande maîtrise de son 
érudition: loin de se borner à faire "l'histoire d'une utopie", il apporte une vision générale de l'histoire culturelle mondiale couvrant la deuxième moitié du XIX ${ }^{e}$ siècle et une bonne partie $d u x^{e}$. On ne peut que souhaiter que ce livre puisse être mis à la portée d'un plus grand nombre de lecteurs francophones à l'occasion d'une traduction.

\section{AUTEURS}

\section{ANNIE PETIT}

Université Montpellier 3 